\title{
Strong Unique Continuation Property for the Dirac Equation
}

\author{
Dedicated to the memory of Professor Nobuhisa Iwasaki
}

By

Laura De CARLI* and Takashi ŌKaJ**

\begin{abstract}
We discuss some Carleman type inequalities which lead to a strong unique continuation property for Dirac operators with potential dominated by the Coulomb singularity.
\end{abstract}

\section{§1. Introduction}

Let $\alpha_{j}, j=1,2,3, \beta=\alpha_{0}$ be anticommuting matrices which satisfy the following relations.

$$
\alpha_{j}^{*}=\alpha_{j}, \quad \alpha_{j}^{2}=I, \quad \alpha_{j} \alpha_{k}+\alpha_{k} \alpha_{j}=0, \quad j \neq k .
$$

The Dirac equation which describes free relativistic electrons is represented by

$$
i \hbar \partial_{t} \psi(t, x)=H_{0} \psi(t, x),
$$

where $H_{0}$ is given explicitly by the $4 \times 4$ matrix-valued differential expression

$$
H_{0}=-i \hbar c \sum_{j=1}^{3} \alpha_{j} \partial_{x_{j}}+\alpha_{0} m c^{2} .
$$

Here, $c$ is the speed of light, $m$ is a mass of a particle and $\hbar$ is the Planck's constant.

More in general, let $\alpha_{j}, j=0, \ldots n$ be $N \times N$ symmetric matrices which form a basis of the Clifford algebra. Namely, they satisfy (1.1). It is known

Communicated by Y. Takahashi, April 9, 1999.

1991 Mathematics Subject Classification(s): 35B05, 35J45, 35R45

* Dipartimento di Matematica e Applicazioni, "Renato Caccioppoli", Università di Napoli, "Federico II", via Cintia 80126 Napoli, Italy

** Department of Mathematics Kyoto Univ., Kyoto, 606-8502, Japan 
that $N$ is in the set $D \mathbf{Z}$, where $D=2^{\left[\frac{n+1}{2}\right]}$. Throughout this paper, we only consider the irreducible cases. Namely, we assume that

$$
N=2^{\left[\frac{n+1}{2}\right]} \text {. }
$$

The (n-dimensional) Dirac operator with which we are concerned is

$$
L=-i \sum_{j=1}^{n} \alpha_{j} \partial_{x_{j}}
$$

which satisfies

$$
L^{2}=-\Delta I
$$

Let $\Omega$ be a domain of $\mathbf{R}^{n}$ containing the origin. We consider two problems for strong unique continuation property. The one concerns the differential inequality

$$
|L u(x)| \leq|V(x) u(x)| \quad x \in \Omega
$$

and the other is about the differential equation

$$
L u(x)+V(x) u(x)=0 \quad x \in \Omega .
$$

In both cases, the singular matrix potential $V$ satisfies

$$
|x| V(x) \in L^{\infty}\left(\mathbf{R}^{n}\right)^{N^{2}} .
$$

There are many works concerning the unique continuation properties for single elliptic equations or for elliptic diagonalizable systems. For general elliptic systems, however, we have only few works (c.f. [5]).

We note first that a simple calculation shows that

$$
\operatorname{det}\left(\lambda-i \sum_{j=1}^{n} \alpha_{j} \xi_{j}\right)=\left\{\begin{array}{lll}
\lambda^{2}+|\xi|^{2}, & \text { if } n=2, & N=2 \\
\left(\lambda^{2}+|\xi|^{2}\right)^{2} & \text { if } n=3, & N=4
\end{array}\right.
$$

The multiplicity of the characteristics becomes higher and higher as the space dimension becomes large. This causes some difficulty in the study for the Dirac operator by using the matrix diagonalization method. Furthermore, the presence of a singular potential makes the problem more complicated. To overcome these difficulties, we shall use eigenfunction expansions. The Dirac operator has solutions corresponding to both positive energy and negative energy. The potential $V$ is assumed to satisfy, roughly speaking, that the multiplication operator $V$ transforms only a few negative states into positive states when the absolute value of energy is high. We shall see that this assumption ensures the strong unique continuation property. We hope that our study throws new light on those problems for general elliptic systems. 
The plan of our paper is as follows. We first state the results for the general space dimension in Section 2. When the space dimension is equal to two or three, we can improve them. The precise results will be stated in Section 3. In Sections 4-6, we shall give their proofs. In the final section, some negative results will be presented.

\section{§2. The General Space Dimension}

Our first result is as follows. Let $W$ be a spherically symmetric scalar potential such that

$$
W(x)=w(|x|) I, \quad|x| w(|x|) \in L^{\infty}\left(\mathbf{R}^{n}\right) .
$$

We denote the operator norm in $\mathbb{R}^{n}$ for the constant matrix $V$ by $\|V\|$.

Theorem 2.1. Let $n \geq 2$ and $\Omega$ be a domain of $\mathbf{R}^{n}$ containing the origin. Suppose $V(x)$ satisfies $|x| V(x) \in L^{\infty}\left(\mathbf{R}^{n}\right)^{N^{2}}$ and

$$
\sup _{x \in \mathbf{R}^{n}}|x|\|V(x)-W(x)\|<1 / 2 .
$$

If $u \in\left\{W_{l o c}^{1,2}(\Omega)\right\}^{N}$ satisfies

$$
L u(x)+V(x) u=0 \quad \text { in } \Omega
$$

and $u$ vanishes of infinite order at the origin, then $u$ is identically zero in $\Omega$.

Remark 2.1. O. Yamada obtained in a paper in preparation this result when $V=W$ and $n=3$.

Here and in what follows, we say that $u \in L_{\text {loc }}^{2}(\Omega)$ vanishes of infinite order at $x_{0} \in \Omega$ if

$$
\lim _{R \rightarrow 0} R^{-\ell} \int_{\left\{\left|x-x_{0}\right|<R\right\}}|u(x)|^{2} d x=0
$$

for every $\ell>0$.

Next, we consider the differential inequality.

Theorem 2.2. Let $n \geq 2$. Let $u \in\left\{W_{\text {loc }}^{1,2}(\Omega)\right\}^{N}$ be a solution of the differential inequality

$$
|L u(x)| \leq \frac{C}{|x|}|u(x)| \quad x \in \Omega,
$$

where $C$ is a positive constant strictly less than $1 / 2$. Then, $u$ is identically zero if it vanishes of infinite order at some point of $\Omega$.

Corollary 2.3. Suppose that there exist positive constants $\varepsilon$ and $C$ such that 


$$
|L u(x)| \leq \frac{C}{|x|^{1-\varepsilon}}|u(x)|, \quad x \in \Omega .
$$

Then, the same conclusion as in Theorem 2.2 holds.

Remark 2.2. V. Vogelsang ([13]) obtained the same result as in Corollary 2.3 when the space dimension is three.

There is also a related results by D. Jerison ([6]) who showed that the differential inequality for the Dirac operator

$$
|L u| \leq|V(x) u|
$$

has the unique continuation property from an open set provided that $L u(x) \in$ $L^{2}(\Omega)^{N}$ and $\|V(x)\| \in L^{\sigma}(\Omega)$ with $\sigma \geq \frac{3 n-2}{2}$.

There are a lot of works on the unique continuation properties for the Laplacean or of a single elliptic equation. In fact, it is known that the differential inequality

$$
|\Delta u(x)| \leq \frac{C_{1}}{|x|^{2}}|u(x)|+\frac{C_{2}}{|x|}|\nabla u(x)|
$$

where $C_{1}$ and $C_{2}$ are positive constants, has the SUCP in $W_{l o c}^{2,2}$ if $C_{2}<\frac{\sqrt{2}}{2}$, ([1], [3], [7], [8], [4], [9], [10]). Moreover, we have counterexamples to SUCP when $n=2$ and $C_{2}>1$, ([2]), and in the higher dimensional case, except for $n=3$, if $C_{2}$ is sufficiently large ([15]).

In view of the relation $\Delta I u=-L L u$, we can translate the negative results for the Laplacean to the cases for the Dirac operator. Especially, when $n=2$, there is a counterexample which shows that the best constant $C$ appearing in Theorem 2.2 should be less than one. See the section 7 for the detail.

Furthermore, our Theorem 2.2 shows that we can take $\|V(x)\| \in L_{l o c}^{n, \infty}(\Omega)$, which is the best possible choice in the scale of the $L^{p}$ spaces. We shall prove this fact at the end of Section 7 using an adaptation of a famous counterexample of D. Jerison and C. Kenig ([7]).

\section{§3. Low Dimensional Cases}

When the space dimension is equal to two or three, we have more precise results. We begin with two dimensional case.

We define Pauli matrices, which are $2 \times 2$, by

$$
\sigma_{1}=\left(\begin{array}{cc}
0 & 1 \\
1 & 0
\end{array}\right), \quad \sigma_{2}=\left(\begin{array}{cc}
0 & -i \\
i & 0
\end{array}\right), \quad \sigma_{3}=\left(\begin{array}{cc}
1 & 0 \\
0 & -1
\end{array}\right) .
$$


The two dimensional Dirac operator is given by

$$
P=-i\left\{\sigma_{1} \partial_{x_{1}}+\sigma_{2} \partial_{x_{2}}\right\}
$$

The potential we consider is a two by two matrix having the following form.

$$
V(x)=\left(\begin{array}{ll}
V_{11}(x) & V_{12}(x) \\
V_{21}(x) & V_{22}(x)
\end{array}\right)
$$

Set

$$
\tilde{V}_{i, j}(x)=|x| V_{i, j}(x) .
$$

Let $T\left(\mathbf{R}_{+} \times \mathbf{S}^{n-1}\right)$ denote the set of all trigonometric polynomials like

$$
\sum_{|k| \leq m} F_{k}(r) e^{i k \theta}, \quad F_{k} \in L^{\infty}\left(\mathbf{R}_{+}\right)
$$

Let $\mathscr{K}$ denote the closure of the subset $T$ in $L^{\infty}\left(\mathbf{R}_{+} \times \mathbf{S}^{n-1}\right)$.

Theorem 3.1. Suppose that each of $\tilde{V}_{j k}(x)$ is bounded in $\mathbf{R}^{2}$,

$$
|x| V_{j j} \in \mathscr{K}, \quad j=1,2,
$$

and

$$
\sup |x|\left|V_{i, j}(x)\right|<\frac{1}{2} \quad \text { if } i+j \text { is odd } .
$$

Then, if $u \in\left\{W_{l o c}^{1,2}(\Omega)\right\}^{2}$ satisfies $P u+V u=0$ and vanishes of infinite order at the origin, $u$ is identically zero.

Corollary 3.2. In place of the condition (3.4), we assume that each of $\tilde{V}_{j j}$ $(j=1,2)$ is continuous in a neighborhood of the origin. Then, the same conclusion as in Theorem 3.1 holds.

We note that Theorem 3.1 does not follow from the result for the Laplacian because the potential is not regular sufficiently.

Next, we turn to the three dimensional case. When $n=3,(1.2)$ implies $N=4$. A basis of the Clifford algebra is given by

$$
\alpha_{0}=\left(\begin{array}{cc}
I & 0 \\
0 & -I
\end{array}\right), \quad \alpha_{j}=\left(\begin{array}{cc}
0 & \sigma_{j} \\
\sigma_{J} & 0
\end{array}\right), \quad j=1,2,3 .
$$

Define the Dirac operator $D$ by

$$
D=-i \sum_{k=1}^{3} \alpha_{j} \partial_{x_{j}}
$$

We consider a $4 \times 4$ matrix potential $V(x)$ with $(i, j)$ component $V_{i j}(x)$. 
We define two subsets $\Delta$ and $\Sigma$ of $\{1,2,3,4\}^{2}$ by

$$
\begin{aligned}
& \Delta=\{(1,1),(1,3),(3,1),(3,3)\}, \\
& \Sigma=\{(i, j):(i, j) \notin \Delta \text { and }(i-1, j-1) \notin \Delta\} .
\end{aligned}
$$

Let $\Omega$ be a domain of $\mathbf{R}^{3}$ containing the origin. Let $W$ be a $4 \times 4$ matrix whose components $W_{i j}$ are spherically symmetric and, moreover, they satisfy

$$
W_{i j}(|x|)=W_{i+1, j+1}(|x|) \quad \text { for }(i, j) \in \Delta
$$

and

$$
|x| W_{i j}(|x|) \in L^{\infty}\left(\mathbf{R}^{3}\right), \quad \forall(i, j)
$$

with

$$
W_{i j} \equiv 0, \quad \text { if }(i, j) \in \Sigma .
$$

Theorem 3.3. Suppose $V$ is a $4 \times 4$ matrix satisfying

$$
\sup |x|\|V(x)-W(x)\|<1 / 2 .
$$

If $u \in\left\{W_{l o c}^{1,2}(\Omega)\right\}^{4}$ satisfies

$$
D u(x)+V(x) u=0 \quad \text { in } \Omega
$$

and vanishes of infinite order at the origin, then $u$ is identically zero in $\Omega$.

\section{§4. The Proofs of Theorems 2.1 and 2.2}

First of all, we recall the fundamental facts about the properties of the Dirac operator (c.f. [6]). Let $n \geq 2$. We shall use the polar coordinates:

$$
r=|x|, \quad \omega=x /|x|, \quad \text { and } \quad y=\log r .
$$

Set $P_{j}=-i \alpha_{j} . \quad P_{j}$ is a skew symmetric matrix. In these coordinates $(y, \omega) \in$ $\mathbf{R} \times \mathbf{S}^{n-1}$, the Dirac operator can be written as follows.

$$
L=e^{-y} A(\omega)\left(\partial_{y} I-G\left(\omega, \partial_{\omega}\right)\right)
$$

where

$$
A(\omega)=\sum_{j=1}^{n} P_{j} \omega_{j}
$$

and

$$
G\left(\omega, \partial_{\omega}\right)=\sum_{h<k} P_{h} P_{k}\left(\omega_{h} \frac{\partial}{\partial \omega_{k}}-\omega_{k} \frac{\partial}{\partial \omega_{h}}\right)
$$


Note that $A$ is a unitary matrix and $A^{*}=-A$. A calculation shows that

$$
\begin{aligned}
-\Delta I & =(A L)^{*} A L \\
& =\left(\partial_{y} I-G\right)^{*} e^{-2 y}\left(\partial_{y} I-G\right) \\
& =\left\{\left(-\partial_{y}-n\right) I-G\right\} e^{-2 y}\left(\partial_{y} I-G\right) \\
& =e^{-2 y}\left\{\left(-\partial_{y}+2-n\right) I-G\right\}\left(\partial_{y} I-G\right) .
\end{aligned}
$$

Since

$$
\Delta=e^{-2 y}\left\{\partial_{y}^{2}+(n-2) \partial_{y}+\Lambda\right\}
$$

where $\Lambda$ is the Laplace-Beltrami operator on the unit sphere $\mathbf{S}^{n-1}$, we have

$$
G(G+n-2)=-\Lambda .
$$

We know that the eigenvalues of $-\Lambda$ are $k(k+n-2), k=0,1,2, \ldots$ Therefore, it turns out that the eigenvalues of $G$ are a subset of $\mathbb{Z}$. In particular, when $n=2$, the spectrum of $G$ is equal to $\mathbf{Z}$. When $n \geq 3$, it coincides with the set $\mathbf{Z} \backslash\{-1,-2, \ldots, 2-n\}$. Let $\mathscr{Q}_{k}$ be the vector space of harmonic $\mathbf{C}^{m}$-valued polynomials of degree $k$, and let $\mathscr{H}_{k}$ be its restriction on $\mathbf{S}^{n-1} . \quad \mathscr{H}_{k}$ is the eigenspace of $-\Lambda I$ that corresponds to the eigenvalue $k(k+n-2)$. In particular, if $E_{k}=\operatorname{ker}(G-k)$, then $E_{k} \oplus E_{2-n-k}=\mathscr{H}_{k}$. Moreover, $E_{-1}=\cdots=E_{2-n}=\{0\}$ when $n \geq 3$.

Now, we establish a crucial relation between $A$ and $G$.

\section{Lemma 4.1.}

$$
A G=(1-n-G) A .
$$

Proof. A simple calculation shows that

$$
A(\omega) G-G A(\omega)=2 A G+(n-1) A .
$$

This implies the conclusion.

Let us define $B=G+\frac{n-1}{2}$. Then,

$$
L=e^{-y} A\left\{\partial_{y}+\frac{n-1}{2}-B\right\} \quad \text { and } \quad A B=-B A .
$$

The set of all the eigenvalues of $B$ is equal to the set $\Sigma=-\left(\mathbf{N}_{0}+\frac{n-1}{2}\right) \cup$ $\left(\mathbf{N}_{0}+\frac{n-1}{2}\right)$, where $\mathbf{N}_{0}=\{0,1,2,3, \ldots\}$. Let $F_{\lambda}$ be the eigenspaces of $B$ corresponding to the eigenvalue $\lambda$ for $\lambda \in \Sigma$. Then, in view of $A^{*} A=I$, we see that $A$ is a bijection from $F_{\lambda}$ to $F_{-\lambda}$ for any $\lambda \in \Sigma$. Let $\left\{u_{\lambda, \ell}\right\}_{\lambda \in \Sigma}$ be the 
orthonormal basis of $L^{2}\left(\mathbf{S}^{n-1}\right)^{N}$ such that

$$
A u_{\lambda, \ell}=u_{-\lambda, \ell}, \quad \ell=1, \ldots, d_{\lambda} .
$$

Here, $d_{\lambda}$ is equal to the dimension of $F_{\lambda}$. Let $\Pi_{\lambda}$ be the-projection on the subspace $F_{\lambda}$ of $L^{2}\left(\mathbf{S}^{1}\right)^{N}$. For an integer $J$, define

$$
\Pi_{J}^{+}=\bigoplus_{\lambda \geq J} \Pi_{\lambda}, \quad \Pi_{J}^{-}=\bigoplus_{\lambda<J} \Pi_{\lambda} .
$$

Summing up, we can conclude that if $u \in L^{2}\left(\mathbb{R}^{n}\right)^{N}$ and

$$
u(r, \omega)=\sum_{\lambda \in \Sigma} \sum_{1 \leq \ell \leq d_{\lambda}} f_{\lambda, \ell}(r) u_{\lambda, \ell}(\omega)
$$

then

$$
L u=e^{-y} \sum_{\lambda \in \Sigma} \sum_{1 \leq \ell \leq d_{\lambda}}\left\{\left(f_{-\lambda, \ell}\right)^{\prime}+\left(\frac{n-1}{2}-\lambda\right) f_{-\lambda, \ell}\right\} u_{\lambda, \ell},
$$

where the prime stands for the derivative in $y=\log r$.

Thus, it holds

$$
L u=e^{-y} \sum_{\lambda \in \Sigma}\left\{\partial_{y}+\left(\frac{n-1}{2}-\lambda\right)\right\} A \Pi_{\lambda} u .
$$

If $u \in C_{0}^{\infty}\left(\mathbb{R}^{n} \backslash\{0\}\right)^{N}$ and $(L+V(x)) u=r^{-1} g$, then (4.2) gives

$$
\left\{\partial_{y}+\left(\frac{n-1}{2}-\lambda\right)\right\} f_{-\lambda, \ell}=-\left(r V(x) u, u_{\lambda, \ell}\right)+\left(g, u_{\lambda, \ell}\right)_{L^{2}\left(\mathbf{S}^{n-1}\right)}
$$

for $\lambda \in \Sigma$. We will show that the equation of (4.3) corresponding to $\lambda$ has a better estimate for the negative $\lambda$ than for the positive $\lambda$ (Lemma 4.3). To utilize this fact, we divide those equations corresponding to the negative $\lambda$ 's by a large positive constant $M$ which will be determined later. This simple trick leads to the condition on the potential $V$ which concerns the interaction of the projection operator $\Pi_{\ell}$ with the multiplication by $V$.

Lemma 4.2. Let $J=0$. Our assumption on the potential implies that there exists a positive constant $C_{0}$ such that

$$
\left\|\Pi_{0}^{+}|x| V(x) u\right\| \leq \varepsilon\left\|\Pi_{0}^{-} u\right\|+C_{0}\left\|\Pi_{0}^{+} u\right\|
$$

with $\varepsilon<1 / 2$.

Proof. In view of the facts that

$$
\left\|\Pi_{0}^{+} r V(x) u\right\| \leq\left\|\Pi_{0}^{+} r V(x) \Pi_{0}^{-} u\right\|+\left\|\Pi_{0}^{+} r V(x) \Pi_{0}^{+} u\right\|
$$

and 


$$
\left\|\Pi_{0}^{+} r W(|x|) \Pi_{0}^{-} u\right\|=0,
$$

the conclusion is an immediate consequence of the condition (3.8).

We require the following Carleman estimate.

Lemma 4.3. For any real $\gamma$, any $f(y) \in C_{0}^{\infty}(\mathbf{R})$ and a real constant $\mu$, we have

$$
\begin{aligned}
\int_{-\infty}^{\infty} e^{-2 \gamma y}\left|\partial_{y} f+\mu f\right|^{2} d y & =\int_{-\infty}^{\infty} e^{-2 \gamma y}\left\{(\gamma+\mu)^{2}|f|^{2}(y)+\left|f^{\prime}\right|^{2}(y)\right\} d y \\
& \geq \int_{-\infty}^{\infty} e^{-2 \gamma y}(\gamma+\mu)^{2}|f|^{2}(y) d y .
\end{aligned}
$$

Proof. Let $v=e^{-\gamma y} f$. Then, the desired identity follows from the facts that

$$
\int_{-\infty}^{\infty} e^{-2 \gamma y}\left|\partial_{y} f+\mu f\right|^{2} d y=\int_{-\infty}^{\infty}\left|\left(\partial_{y}+\gamma+\mu\right) v\right|^{2} d y
$$

and that

$$
2 \Re \int_{-\infty}^{\infty}(\gamma+\mu) v(y) \overline{v^{\prime}}(y) d y=0 .
$$

We divide each of the equations (4.3) with $\lambda<0$ by a sufficiently large positive number $M$ which will be determined later. An application of this lemma to the new system obtained from (4.3), together with Lemma 4.2 with $\mu=\mu_{\lambda}=(n-1) / 2-\lambda \in \mathbf{Z}$ implies that there exist some $0<\tilde{\varepsilon}<1 / 2$ and positive constants $C_{j}, j=0,1,2$ such that

$$
\begin{aligned}
& \sum_{\lambda \in \Sigma, \lambda>0}\left(\gamma+\mu_{\lambda}\right)^{2} \int e^{-2 y \gamma}\left\|\Pi_{-\lambda} u\right\|^{2} d y \\
& \quad \leq \int e^{-2 \gamma y}\left\|\Pi_{0}^{+}(-r V(x) u+g)\right\|^{2} d y \\
& \quad \leq \tilde{\varepsilon}^{2} \int e^{-2 \gamma y}\left\|\Pi_{0}^{-} u\right\|^{2} d y+\tilde{C}_{0} \int e^{-2 \gamma y}\left\|\Pi_{0}^{+} u\right\|^{2} d y+C \int e^{-2 \gamma y}\|g\|^{2} d y
\end{aligned}
$$

and

$$
\begin{aligned}
& \frac{1}{M} \sum_{\lambda \in \Sigma, \lambda \leq 0}\left(\gamma+\mu_{\lambda}\right)^{2} \int e^{-2 \gamma y}\left\|\Pi_{-\lambda} u\right\|^{2} d y \\
& \quad \leq \frac{1}{M} \int e^{-2 \gamma y}\left\|\Pi_{0}^{-}(-r V(x) u+g)\right\|^{2} d y \\
& \quad \leq \frac{1}{M} \int e^{-2 \gamma y}\left\{C_{1}\left\|\Pi_{0}^{+} u\right\|^{2}+C_{2}\left\|\Pi_{0}^{-} u\right\|^{2}+\|g\|^{2}\right\} d y .
\end{aligned}
$$


Here, we have used the inequality

$$
(a+b)^{2} \leq \delta a^{2}+\frac{\delta}{\delta-1} b^{2} \quad \text { for } a, b>0, \delta>1 .
$$

Note that if $\lambda \leq 0$, then $\left|\gamma+\mu_{\lambda}\right| \geq \gamma$ for any $\gamma>1$. On the other hand, if $\lambda>0$, then $\left|\gamma+\mu_{\lambda}\right| \geq 1 / 2$ for any $\gamma \in \mathbb{N}+1 / 2$ since $\mu_{\lambda} \in \mathbb{Z}$. Therefore, taking both $M>0$ and $\gamma \in \mathbf{N}+1 / 2$ to be large enough, we can conclude that

$$
\begin{gathered}
\left(\frac{1}{4}-\varepsilon^{2}-C_{2} / M\right)^{1 / 2}\left\{\sum_{\lambda \in \Sigma} \sum_{\ell=1}^{d_{\lambda}} \int e^{-2 \gamma y}\left|f_{-\lambda, \ell}\right|^{2} d y\right\}^{1 / 2} \\
\leq C\left\{\int e^{-2 \gamma y}\|g\|_{L^{2}\left(\mathbf{S}^{n-1}\right)}^{2} d y\right\}^{1 / 2} .
\end{gathered}
$$

Now, we are in a position to prove Theorem 2.1. Choose $\varphi \in C^{\infty}(\mathbf{R})$ such that

$$
\begin{gathered}
0 \leq \varphi(t) \leq 1 \\
\varphi(y)= \begin{cases}1, & \text { if } y \geq-1 \\
0, & \text { if } y \leq-3\end{cases}
\end{gathered}
$$

Define $\chi \in C^{\infty}(\mathbf{R})$ by

$$
\chi(y)=1 \quad \text { if } y \leq-3 \text { and }=0 \quad \text { if } y \geq-2 .
$$

Let $u \in W_{l o c}^{1,2}(\Omega)^{N}$ be a solution to $L u+V u=0$ and assume that it vanishes of infinite order at the origin. Set

$$
U_{j}(y, \omega)=\varphi(y / j) \chi(y / R) u\left(e^{y}, \omega\right) .
$$

Here, we take $R$ to be a large positive number such that the support of $U_{j}(\log |x|, \omega)$ is a compact set of $\Omega$. The standard limiting argument implies that the Carleman inequality (4.7) is still valid for $U_{j}$. Applying the inequality (4.7) to $U_{j}$, we see that there exists a positive constant $\gamma_{0}$ such that for any $\gamma>\gamma_{0}$ in $\mathbf{N}+1 / 2$,

$$
\begin{aligned}
\int e^{-2 y \gamma}\left|U_{j}(y, \omega)\right|^{2} d y d \omega \\
\leq C \int_{-3 j}^{\infty} d y \int e^{-2 y \gamma}\left|j^{-1} \varphi^{\prime}(y / j) \chi(y / R) u\right|^{2} d \omega \\
\quad+C_{R} \int_{-\infty}^{-2 R} d y \int e^{-2 y \gamma}\left|\chi^{\prime}(y / R) u\right|^{2} d \omega .
\end{aligned}
$$


Let $j \rightarrow \infty$. Then, from the hypothesis that $u$ vanishes of infinite order at the origin, we see that for any fixed $\gamma>1$

$$
\begin{aligned}
& \lim _{j \rightarrow \infty} \int_{y>-3 j} \int_{\mathbf{S}^{n-1}} e^{-2 y \gamma}\left|u\left(e^{y}, \omega\right)\right|^{2} d y d \omega \\
& \quad \leq \lim _{j \rightarrow \infty} e^{6 j(\gamma+n / 2)} \int_{y>-3 j} \int_{\mathbf{S}^{n-1}}\left|u\left(e^{y}, \omega\right)\right|^{2} e^{n y} d y d \omega=0,
\end{aligned}
$$

since there exists a positive integer $\ell$ such that $\ell \leq \gamma+n / 2<\ell+1$ and $e^{6 j(\gamma+n / 2)}$ $\leq\left\{e^{3 j}\right\}^{2 \ell+2}$. As a result, we obtain that

$$
\int_{|x| \leq e^{-4 R}}|x|^{-2 \gamma-n}|u(|x|, \omega)|^{2} d x \leq \tilde{C}_{R} \int_{|x| \geq e^{-3 R}}|x|^{-2 \gamma-n}|u(|x|, \omega)|^{2} d x .
$$

Thus, it holds that

$$
e^{4 R(\gamma+n / 2)} \int_{|x| \leq e^{-4 R}}|u|^{2} d x \leq \tilde{C}_{R} e^{3 R(\gamma+n / 2)} \int_{|x| \leq e^{-3 R}}|u|^{2} d x .
$$

Dividing both sides of the above inequality by $e^{3 R(\gamma+n / 2)}$ and letting $\gamma \rightarrow \infty$, we have

$$
u(x)=0 \quad \text { on }|x| \leq e^{-4 R} .
$$

This completes the proof of Theorem 2.1.

Now, we turn to the proof of Theorem 2.2. The analogous argument to that in the preceding proof yields the following Carleman-type estimate.

Theorem 4.4. Let $n \geq 2$ and let $r=|x|$. There exists a positive number $\gamma_{0}$ such that

$$
\frac{1}{2}\left\|r^{-\gamma-1} u\right\|_{L^{2}} \leq\left\|r^{-\gamma} L u\right\|_{L^{2}}
$$

holds for every $u \in C_{0}^{\infty}\left(\mathbf{R}^{n} \backslash\{0\}\right)^{N}$ and $\gamma>\gamma_{0}$ in $\mathbf{N}$ or $\mathbf{N}+/ 2$ if $n$ is odd or even, respectively.

Proof. Keep the same notation in the preceding section. Since

$$
\int_{\mathbf{S}^{n-1}}|u(y, \omega)|^{2} d \omega=\sum_{\lambda \in \Sigma} \sum_{1 \leq \ell \leq d_{\lambda}}\left|f_{\lambda, \ell}(y)\right|^{2},
$$

an application of Lemma 4.3 to the system (4.3) with $V=0$ yields the conclusion of Theorem 4.4 because of

$$
\left\||x|^{-\gamma} u\right\|_{L^{2}\left(\mathbf{R}^{n}\right)^{N}}^{2}=\int_{-\infty}^{\infty} e^{-(2 \gamma-n) y}\|u\|_{L^{2}\left(\mathbf{S}^{n-1}\right)^{N}}^{2} d y .
$$


In view of Theorem 4.4, the proof of Theorem 2.2 follows from the standard argument.

\section{$\S 5$. The Proof for the Two Dimensional Case}

For the two dimensional case, we can obtain precise information on the eigenfunction of the angular part of the Dirac operator. Let us introduce the polar coordinates $x=e^{y} \omega$, i.e, $y=\log |x|$ and $\omega=\frac{x}{|x|}=(\cos \theta, \sin \theta)$. Define an orthogonal matrix $A$ by

$$
A=\sigma_{1} \omega_{1}+\sigma_{2} \omega_{2}=\left(\begin{array}{cc}
0 & e^{-i \theta} \\
e^{i \theta} & 0
\end{array}\right) .
$$

$P$ can be represented by

$$
P=-i e^{-y}\left(\left(\sigma_{1} \omega_{1}+\sigma_{2} \omega_{2}\right) \frac{\partial}{\partial y}+\left(\sigma_{1} \frac{\partial}{\partial \omega_{1}}+\sigma_{2} \frac{\partial}{\partial \omega_{2}}\right)\right) .
$$

Since $A^{2}=I$, the last expression is equal to

$$
-i e^{-y} A\left(\frac{\partial}{\partial y} I_{2}+\left(\begin{array}{cc}
i & 0 \\
0 & -i
\end{array}\right) \frac{\partial}{\partial \theta}\right)
$$

where we have used the relation

$$
\sigma_{1} \sigma_{2}\left(\omega_{1} \frac{\partial}{\partial \omega_{2}}-\omega_{2} \frac{\partial}{\partial \omega_{1}}\right)=\left(\begin{array}{cc}
i & 0 \\
0 & -i
\end{array}\right) \frac{\partial}{\partial \theta}
$$

In what follows, we denote

$$
B=\left(\begin{array}{cc}
i & 0 \\
0 & -i
\end{array}\right) \quad \text { and } \quad G=\left(\begin{array}{cc}
i & 0 \\
0 & -i
\end{array}\right) \frac{\partial}{\partial \theta} .
$$

The equation $P u+V(x) u=0$ reduces to

$$
-i A\left(\frac{\partial}{\partial y} I+G\right) u+\tilde{V} u=0 .
$$

Here,

$$
\tilde{V}(y, \theta)=e^{y} V\left(e^{y} \cos \theta, e^{y} \sin \theta\right) .
$$

For each positive integer $k$, define the subspaces $E_{k}^{ \pm}$of $L^{2}\left(\mathbb{S}^{1}\right)^{2}$ by

$$
E_{k}^{+}=\left\{\left(\begin{array}{c}
c_{1} e^{-i k \theta} \\
c_{2} e^{i k \theta}
\end{array}\right): c_{\jmath} \in \mathbb{C}\right\} \quad \text { and } \quad E_{k}^{-}=\left\{\left(\begin{array}{c}
c_{1} e^{i(k-1) \theta} \\
c_{2} e^{-i(k-1) \theta}
\end{array}\right): c_{j} \in \mathbb{C}\right\} .
$$


It is easily verified that $E_{k}^{ \pm}$is the eigenspace of $G-1 / 2$ corresponding to the eigenvalue $\pm(k-1 / 2)$. Moreover,

$$
A\left(\begin{array}{c}
c_{1} e^{-\imath k \theta} \\
c_{2} e^{i k \theta}
\end{array}\right)=\left(\begin{array}{c}
c_{2} e^{i(k-1) \theta} \\
c_{1} e^{-i(k-1) \theta}
\end{array}\right) .
$$

We shall use the following notation.

$$
\tilde{V}(y, \theta)=\left(\begin{array}{cc}
\tilde{V}_{+}(x) & \tilde{V}_{1}(x) \\
\tilde{V}_{2}(x) & \tilde{V}_{-}(x)
\end{array}\right)
$$

Let $Q_{k}$ be the projection on the subspace spanned by $e^{-i k \theta}$ of $L^{2}\left(\mathbf{S}^{1}\right)$. Define

$$
Q_{J}^{+}=\bigoplus_{k \geq J} Q_{k}, \quad Q_{J}^{-}=\bigoplus_{k<J} Q_{k}
$$

When the space dimension is two, the condition (4.4) can be translated as follows. We assume that the potential $V$ satisfies that there exist a nonnegative integer $J$ and two positive constants $\varepsilon, \varepsilon^{\prime}$ and a positive constant $C$ such that $\varepsilon+\varepsilon^{\prime}<1 / 2$

$$
\begin{gathered}
\left\|Q_{J}^{+} \tilde{V}_{+} Q_{-J+1}^{-} u\right\| \leq \varepsilon\left\|Q_{-J+1}^{-} u\right\|+C\left\|Q_{-J+1}^{+} u\right\| \\
\left\|Q_{-J}^{-} \tilde{V}_{-} Q_{J+1}^{+} u\right\| \leq \varepsilon\left\|Q_{J+1}^{+} u\right\|+C\left\|Q_{J+1}^{-} u\right\|
\end{gathered}
$$

and

$$
\begin{gathered}
\left\|Q_{J}^{+} \tilde{V}_{1} Q_{J+1}^{+} u\right\| \leq \varepsilon^{\prime}\left\|Q_{J+1}^{+}\right\|+C\left\|Q_{J+1}^{-} u\right\| \\
\left\|Q_{-J}^{-} \tilde{V}_{2} Q_{-J+1}^{-} u\right\| \leq \varepsilon^{\prime}\left\|Q_{-J+1}^{-} u\right\|+C\left\|Q_{-J+1}^{+} u\right\|
\end{gathered}
$$

where the norm stands for the standard operator norm in $L^{2}\left(\mathbf{S}^{n-1}\right)$.

First of all, we prove the following result.

Theorem 5.1. Suppose the conditions (5.2) and (5.3) hold. Then, if Pu+ $V u=0$ and $u$ vanishes of infinite order at the origin, then, $u$ is identically zero.

Now, we proceed to prove this theorem. We shall decompose $u(y, \theta)$ into the sum of Fourier series. Recalling that $L^{2}\left(\mathbf{S}^{1}\right)=\bigoplus_{k=-\infty}^{+\infty} \mathbf{C} e^{-i k \theta}$, we can write

$$
u(y, \theta)={ }^{t}\left(u^{+}, u^{-}\right)=\sum_{k \in \mathbf{Z}}\left(\begin{array}{c}
u_{k}^{+}(y) \\
u_{k}^{-}(y)
\end{array}\right) e^{-i k \theta}
$$

where

$$
u_{k}^{ \pm}(y) \in L^{2}\left(-\infty,+\infty ; e^{n y} d y\right)
$$


It follows that

$$
\begin{aligned}
& -i A\left\{\partial_{y}+B \partial_{\theta}\right\} u(y, \theta)+\tilde{V}(y) u(y, \theta) \\
& \quad=\sum_{k \in \mathbf{Z}}\left(\begin{array}{c}
-i\left(\partial_{y}-k-1\right) u_{k+1}^{-}(y)+\left(\tilde{V}_{+}(y) u^{+}\right)_{k}(y)+\left(\tilde{V}_{1}(y) u^{-}\right)_{k}(y) \\
-i\left(\partial_{y}+k+1\right) u_{k+1}^{+}(y)+\left(\tilde{V}_{-}(y) u_{-}\right)_{k}(y)+\left(\tilde{V}_{2}(y) u^{-}\right)_{k}(y)
\end{array}\right) e^{-i k \theta}
\end{aligned}
$$

Here, $(f)_{k}$ stands for the $k$-th Fourier coefficient of $f(\theta)$ :

$$
(f)_{k}(y)=\int f(y, \theta) e^{i k \theta} d \theta
$$

Set $g={ }^{t}\left(g^{+}, g^{-}\right)$. Therefore, $P u+V u=e^{-y} g$ implies

$$
\begin{aligned}
& \sum_{k \in \mathbf{Z}}\left\{-i\left(\partial_{y}-k-1\right) u_{k+1}^{-}(y)+\left(\tilde{V}_{+}(y) u^{+}\right)_{k}(y)+\left(\tilde{V}_{1}(y) u^{-}\right)_{k}(y)\right\} e^{-i k \theta}=g^{+} \\
& \sum_{k \in \mathbf{Z}}\left\{-i\left(\partial_{y}+k+1\right) u_{k+1}^{+}(y)+\left(\tilde{V}_{-}(y) u_{-}\right)_{k}(y)+\left(\tilde{V}_{2}(y) u^{-}\right)_{k}(y)\right\} e^{-i k \theta}=g^{-}
\end{aligned}
$$

Let $J$ be a large positive number for which the condition (5.2) and (5.3) hold. We divide each of the above summations into two parts:

$$
\begin{aligned}
& \sum_{k \geq J}-i\left(\partial_{y}-k-1\right) u_{k+1}^{-}(y) e^{-i k \theta}=-Q_{J}^{+}\left(\tilde{V}_{+}(y) u^{+}+\tilde{V}_{1}(y) u^{-}-g^{+}\right) \\
& \sum_{k>-J}-i\left(\partial_{y}+k-1\right) u_{-k+1}^{-}(y) e^{i k \theta}=-Q_{J}^{-}\left(\tilde{V}_{+}(y) u^{+}+\tilde{V}_{1}(y) u^{-}-g^{+}\right)
\end{aligned}
$$

and

$$
\begin{aligned}
\sum_{k \geq-J}-i\left(\partial_{y}+k+1\right) u_{k+1}^{+}(y) e^{-i k \theta} & =-Q_{-J}^{+}\left(\tilde{V}_{-}(y) u_{-}+\tilde{V}_{2}(y) u^{-}-g^{-}\right) \\
\sum_{k>J}-i\left(\partial_{y}-k+1\right) u_{-k+1}^{+}(y) e^{i k \theta} & =-Q_{-J}^{-}\left(\tilde{V}_{-}(y) u_{-}+\tilde{V}_{2}(y) u^{-}-g^{-}\right)
\end{aligned}
$$

Applying Lemma 4.3 to each left hand side of this system, we get

$$
\begin{gathered}
\sum_{k \geq J} \int_{-\infty}^{\infty} e^{-2 \gamma y}\left|\left(\partial_{y}-k-1\right) u_{k+1}^{-}\right|^{2} d y \geq \frac{1}{4}\left\|e^{-\gamma y} Q_{J+1}^{+} u^{-}\right\|^{2}, \\
\sum_{k>-J} \int_{-\infty}^{\infty} e^{-2 \gamma y}\left|\left(\partial_{y}+k-1\right) u_{-k+1}^{-}\right|^{2} d y \geq(\gamma-J-1)^{2}\left\|e^{-\gamma y} Q_{J+1}^{-} u^{-}\right\|^{2}, \\
\sum_{k \geq-J} \int_{-\infty}^{\infty} e^{-2 \gamma y}\left|\left(\partial_{y}+k+1\right) u_{k+1}^{+}\right|^{2} d y \geq(\gamma-J+1)^{2}\left\|e^{-\gamma y} Q_{-J+1}^{+} u^{+}\right\|^{2},
\end{gathered}
$$

and finally 


$$
\sum_{k>J} \int_{-\infty}^{\infty} e^{-2 \gamma y}\left|\left(\partial_{y}-k+1\right) u_{-k+1}^{+}\right|^{2} d y \geq \frac{1}{4}\left\|e^{-\gamma y} Q_{-J+1}^{-} u^{+}\right\|^{2}
$$

for any $f(y) \in C_{0}^{\infty}(\mathbf{R})$ if $\gamma \in \mathbf{N}+\frac{1}{2}$ is greater than $J+1$. Let $M$ be another positive large constant which will be determined later. By virtue of (5.7) and (5.8), we get

$$
\begin{gathered}
\frac{1}{2}\left\|e^{-\gamma y} Q_{J+1}^{+} u^{-}\right\| \leq\left\|Q_{J}^{+}\left(\tilde{V}_{+} u^{+}+\tilde{V}_{1} u^{-}-g^{+}\right)\right\| \\
\frac{\gamma-J-1}{M}\left\|e^{-\gamma y} Q_{J+1}^{-} u^{-}\right\| \leq \frac{1}{M}\left\|Q_{J}^{-}\left(\tilde{V}_{+} u^{+}+\tilde{V}_{1} u^{-}-g^{+}\right)\right\|, \\
\frac{\gamma-J+1}{M}\left\|e^{-\gamma y} Q_{-J+1}^{+} u^{+}\right\| \leq \frac{1}{M}\left\|Q_{-J}^{+}\left(\tilde{V}_{2} u^{+}+\tilde{V}_{-} u^{-}-g^{-}\right)\right\|,
\end{gathered}
$$

and

$$
\frac{1}{2}\left\|e^{-\gamma y} Q_{-J+1}^{-} u^{+}\right\| \leq\left\|Q_{-J}^{-}\left(\tilde{V}_{2} u^{+}+\tilde{V}_{-} u^{-}-g^{-}\right)\right\| .
$$

Our assumption implies that

$$
\begin{aligned}
\left\|Q_{J}^{+}\left(\tilde{V}_{+} u^{+}+\tilde{V}_{1} u^{-}-g^{+}\right)\right\| \leq & \varepsilon_{1}\left\|Q_{-J+1}^{-} u^{+}\right\|+C_{1}\left\|Q_{-J+1}^{+} u^{+}\right\| \\
& +\delta_{1}\left\|Q_{J+1}^{+} u^{-}\right\|+C_{2}\left\|Q_{J+1}^{-} u^{-}\right\| \\
& +C_{3}\left\|g^{+}\right\|
\end{aligned}
$$

and

$$
\begin{aligned}
\left\|Q_{-J}^{-}\left(\tilde{V}_{2} u^{+}+\tilde{V}_{-} u^{-}-g^{-}\right)\right\| \leq & \varepsilon_{2}\left\|Q_{-J+1}^{-} u^{+}\right\|+C_{1}\left\|Q_{-J+1}^{+} u^{+}\right\| \\
& +\delta_{2}\left\|Q_{J+1}^{+} u^{-}\right\|+C_{2}\left\|Q_{J+1}^{-} u^{-}\right\| \\
& +C_{3}\left\|g^{-}\right\|
\end{aligned}
$$

with

$$
\varepsilon_{1}+\varepsilon_{2}<1 / 2, \quad \delta_{1}+\delta_{2}<1 / 2 .
$$

Here, we have used the fact that

$$
\left\|Q_{-J}^{-} \tilde{V}_{2} u^{+}\right\| \leq \sup \left|\tilde{V}_{2}\right|\left\|u^{+}\right\|
$$

and etc..

Taking $M$ to be large enough, we have

$$
\begin{aligned}
\frac{1}{M}\left\|Q_{J}^{+}\left(\tilde{V}_{+} u^{+}+\tilde{V}_{1} u^{-}-g^{+}\right)\right\| \leq & \varepsilon_{3}\left\{\left\|Q_{-J+1}^{-} u^{+}\right\|+\left\|Q_{-J+1}^{+} u^{+}\right\|\right\} \\
& +\delta_{3}\left\{\left\|Q_{J+1}^{+} u^{-}\right\|+\left\|Q_{J+1}^{-} u^{-}\right\|\right\} \\
& +C_{4}\left\|g^{+}\right\|
\end{aligned}
$$




$$
\begin{aligned}
\frac{1}{M}\left\|Q_{-J}^{-}\left(\tilde{V}_{2} u^{+}+\tilde{V}_{-} u^{-}-g^{-}\right)\right\| \leq & \varepsilon_{3}\left\{\left\|Q_{-J+1}^{-} u^{+}\right\|+\left\|Q_{-J+1}^{+} u^{+}\right\|\right\} \\
& +\delta_{3}\left\{\left\|Q_{J+1}^{+} u^{-}\right\|+\left\|Q_{J+1}^{-} u^{-}\right\|\right\} \\
& +C_{4}\left\|g^{-}\right\|
\end{aligned}
$$

with

$$
\varepsilon_{1}+\varepsilon_{2}+\varepsilon_{3}<1 / 2, \quad \delta_{1}+\delta_{2}+\delta_{3}<1 / 2 .
$$

Therefore, we can conclude

Lemma 5.2. There exists a positive constant $C$ such that if $U \in$ $\left\{C_{0}^{\infty}\left(\mathbf{R}^{2} \backslash\{0\}\right)\right\}^{2}$, then

$$
\int|x|^{-2 \gamma}|P U+V U|^{2} d x \geq C \int|x|^{-2 \gamma-2}|U|^{2} d x
$$

for any large $\gamma \in \mathbf{N}+\frac{1}{2}$.

The standard argument leads to the conclusion of Theorem 5.1.

Now, we are in a position to prove Theorem 3.1. What we have to prove is the following result.

Lemma 5.3. Suppose that $W(x) \in \mathscr{K}$ defined in $\S 3$. For any positive number $\varepsilon$, there exist a positive integer $J$ and a constant $C$ such that

$$
\left\|Q_{J}^{+} W(x) Q_{-J+1}^{-} u\right\| \leq \varepsilon\left\|Q_{-J+1}^{-} u\right\|+C\left\|Q_{-J+1}^{+} u\right\|
$$

and

$$
\left\|Q_{-J}^{-} W(x) Q_{J+1}^{+} u\right\| \leq \varepsilon\left\|Q_{J+1}^{+} u\right\|+C\left\|Q_{J+1}^{-} u\right\|
$$

for all $u \in L^{2}\left(\mathbf{R}^{2}\right)$.

Proof. Let $\varepsilon>0$ be given. One can find a trigonometric polynomial $W_{N}$ such that

$$
\begin{aligned}
R_{N}\left(e^{y}, \theta\right) & =W\left(e^{y}, \theta\right)-W_{N}, \\
W_{N} & =\sum_{|k| \leq N} f_{k}\left(e^{y}\right) e^{-i k \theta}
\end{aligned}
$$

and

$$
\left\|R_{N}\right\|_{L^{\infty}\left(\mathbf{R} \times \mathbf{S}^{1}\right)} \leq \varepsilon / 2 .
$$

It holds that

$$
\left\|Q_{J}^{+} W(x) Q_{-J+1}^{-} u\right\| \leq \frac{\varepsilon}{2}\left\|Q_{-J+1}^{-} u\right\|+\left\|Q_{J}^{+} W_{N} Q_{-J+1}^{-} u\right\| .
$$


If we take $J>2 N$, we get

$$
Q_{J}^{+} W_{N} Q_{-J+1}^{-} u=0 .
$$

This completes the proof of Theorem 3.1.

\section{§6. The Proof for the Three Dimensional Case}

We borrow some facts concerning eigenfunctions for the three dimensional Dirac operator from B. Thaller's book $(\S 4.6 .4)$. In what follows, $\beta$ stands for the $4 \times 4$ matrix

$$
\beta=\left(\begin{array}{cc}
I & 0 \\
0 & -I
\end{array}\right)
$$

With the same notation as in Section 4, the orthonormal basis $\left\{\Phi_{k, \pm}^{m}\right\}_{m \in \mathbf{Z},|m| \leq|k|}$ of each eigenspace $E_{k}$ corresponding to the eigenvalue $k \in \mathbf{Z}$ of the operator $\beta(G+1)$ is described as follows. For each nonnegative integer $k$, define

$$
\Phi_{k,+}^{m}=\left(\begin{array}{c}
i \psi_{k,-}^{m} \\
0
\end{array}\right), \quad \Phi_{k,-}^{m}=\left(\begin{array}{c}
0 \\
\psi_{k,+}^{m}
\end{array}\right), \quad \text { for } m=-k-1,-k, \ldots, k, k+1
$$

and

$\Phi_{-k,+}^{m}=\left(\begin{array}{c}i \psi_{k,+}^{m} \\ 0\end{array}\right), \quad \Phi_{-k,-}^{m}=\left(\begin{array}{c}0 \\ \psi_{k,-}^{m}\end{array}\right), \quad$ for $m=-k-1,-k, \ldots, k, k+1$.

Then, it holds

$$
\beta(G+1) \Phi_{n, \pm}^{m}=-n \Phi_{n, \pm}^{m}
$$

Here,

$$
\psi_{k,+}^{m}=\left(\begin{array}{c}
\mu_{k, m}^{+} Y_{k}^{m} \\
-v_{k, m}^{+} Y_{k}^{m+1}
\end{array}\right) \quad \text { and } \quad \psi_{k,-}^{m}=\left(\begin{array}{c}
\mu_{k, m}^{-} Y_{k-1}^{m} \\
v_{k, m}^{-} Y_{k-1}^{m+1}
\end{array}\right)
$$

with nonnegative constants $\mu_{k, m}^{ \pm}$and $v_{k, m}^{ \pm}$whose explicit forms are given by

$$
\begin{array}{llrl}
\mu_{k, m}^{-} & =\sqrt{k+m} / \sqrt{2 k-1}, & v_{k, m}^{-} & =\sqrt{k-m-1} / \sqrt{2 k-1} \\
\mu_{k, m}^{+} & =\sqrt{k-m} / \sqrt{2 k+1}, & v_{k, m}^{+} & =\sqrt{k+m+1} / \sqrt{2 k+1} .
\end{array}
$$

$Y_{k}^{m}(\omega)$ are the spherical harmonics of order $k$ which form a complete orthonormal set in $L^{2}\left(\mathbf{S}^{2}\right)$ : Using the polar coordinates $(r, \theta, \varphi)$ such that the $x_{3}$ axis corresponds to $\varphi=0$, they are given by

$$
Y_{k}^{m}(\theta, \varphi)=\sqrt{\frac{2 k+1(k-m) !}{4 \pi(k+m) !}} e^{i m \varphi} P_{k}^{m}(\cos \theta), \quad \text { for } k \geq m>0,
$$


where

$$
P_{k}^{m}(x)=\frac{(-1)^{m}}{2^{k} k !}\left(1-x^{2}\right)^{m / 2} \frac{d^{m+k}}{d x^{m+k}}\left(x^{2}-1\right)^{k} \quad \text { and } \quad Y_{k}^{-m}=(-1)^{m} \overline{Y_{k}^{m}}
$$

for $|m| \leq k$ and define $Y_{k}^{m}=0$ if $|m|>k$. Moreover, it holds that

$$
\left\{L^{2}\left(\mathbf{S}^{2}\right)\right\}^{4}=\bigoplus_{k \in \mathbf{Z}} E_{k}
$$

and

$$
i A\left(\begin{array}{c}
\psi_{k, \pm}^{m} \\
0
\end{array}\right)=\left(\begin{array}{c}
0 \\
\psi_{k, \mp}^{m}
\end{array}\right) \quad \text { and } \quad i A\left(\begin{array}{c}
0 \\
\psi_{k, \pm}^{m}
\end{array}\right)=\left(\begin{array}{c}
\psi_{k, \mp}^{m} \\
0
\end{array}\right)
$$

These last expressions imply that the multiplication by $A(\omega)|x| V(|x|)$ operates invariantly on the sum of the two eigenspaces corresponding to two eigenvalues having the same absolute value.

We split each of $u \in\left(L^{2}\left(\mathbf{R} \times \mathbf{S}^{2}\right)\right)^{4}$ and $L u=h$ into two component,

$$
u=\left(\begin{array}{l}
u^{+} \\
u^{-}
\end{array}\right) \quad \text { and } \quad h=\left(\begin{array}{l}
h^{+} \\
h^{-}
\end{array}\right)
$$

where each of $u^{ \pm}(y, \cdot)$ belongs to $\left(L^{2}\left(\mathbf{S}^{2}\right)\right)^{2}$ and can be represented as the partial wave expansion:

$$
\begin{aligned}
& u^{+}(y, \omega)=\sum_{n=0}^{\infty} \sum_{m=-n}^{n}\left(f_{n, m}^{+}(y) \psi_{n,+}^{m}(\omega)+f_{n, m}^{-}(y) \psi_{n,-}^{m}(\omega)\right) \\
& u^{-}(y, \omega)=\sum_{n=0}^{\infty} \sum_{m=-n}^{n}\left(g_{n, m}^{+}(y) \psi_{n,+}^{m}(\omega)+g_{n, m}^{-}(y) \psi_{n,-}^{m}(\omega)\right) .
\end{aligned}
$$

Then,

$$
\int_{\mathbf{S}^{2}}\left|u^{+}\right|^{2} d \omega=\sum_{n=0}^{\infty} \sum_{m=-n}^{n}\left(\left|f_{n, m}^{+}\right|^{2}+\left|f_{n, m}^{-}\right|^{2}\right)
$$

and the analogous formula for $u^{-}$holds.

Since

$$
L u=-i e^{-y} A\left(\partial_{y}+1-(G+1)\right) u=e^{-y} h,
$$

(6.1) and (6.4) imply that

$$
-\sum_{n, m}\left\{\left(\partial_{y}+n+1\right) f_{n, m}^{+}\left(\begin{array}{c}
0 \\
\psi_{n,-}^{m}
\end{array}\right)+\left(\partial_{y}-n+1\right) f_{n, m}^{-}\left(\begin{array}{c}
0 \\
\psi_{n,+}^{m}
\end{array}\right)\right\}=\left(\begin{array}{c}
0 \\
h^{-}
\end{array}\right)
$$

and

$$
-\sum_{n, m}\left\{\left(\partial_{y}+n+1\right) g_{n, m}^{+}\left(\begin{array}{c}
\psi_{n,-}^{m} \\
0
\end{array}\right)+\left(\partial_{y}-n+1\right) g_{n, m}^{-}\left(\begin{array}{c}
\psi_{n,+}^{m} \\
0
\end{array}\right)\right\}=\left(\begin{array}{c}
h^{+} \\
0
\end{array}\right) .
$$


Denote by $\Pi_{n}^{+}$a projection onto the subspaces $E_{n}^{+}$spanned by $\left\{\psi_{n,+}^{m}\right\}_{m=-n-1}^{n+1}$ and define $\Pi_{n}^{-}$similarly. Set

$$
P_{k}= \begin{cases}\Pi_{k}^{+} & \text {if } k \geq 0 \\ \Pi_{k}^{-} & \text {if } k<0\end{cases}
$$

and

$$
P_{J}^{+}=\bigoplus_{k \geq J} P_{k} \quad \text { and } \quad P_{J}^{-}=\bigoplus_{k<J} P_{k}
$$

for an integer $J$.

Split $V$ into the $2 \times 2$ blocks as follows:

$$
|x| V(x)=\left(\begin{array}{cc}
V_{+} & V_{1} \\
V_{2} & V_{-}
\end{array}\right) .
$$

Let $L u+V u=g$. Then, we have

$$
h^{+}=-V_{+} u^{+}-V_{1} u^{-}+g^{+} \quad \text { and } \quad h^{-}=-V_{2} u^{+}-V_{-} u^{-}+g^{-} .
$$

The assumption (3.7) on the potential enables us to prove the following result.

Proposition 6.1. There exists a positive large integer $J$ such that

$$
\begin{gathered}
\left\|P_{J}^{+} V_{+} u^{+}\right\| \leq \varepsilon\left\|P_{-J}^{-} u^{+}\right\|+C\left\|P_{-J}^{+} u^{+}\right\| \\
\left\|P_{J}^{+} V_{-} u^{-}\right\| \leq \varepsilon\left\|P_{-J}^{-} u^{-}\right\|+C\left\|P_{-J}^{+} u^{-}\right\| \\
\left\|P_{J}^{+} V_{1} u^{-}\right\| \leq \varepsilon^{\prime}\left\|P_{-J}^{-} u^{-}\right\|+C\left\|P_{-J}^{+} u^{-}\right\| \\
\left\|P_{J}^{+} V_{2} u^{+}\right\| \leq \varepsilon^{\prime}\left\|P_{-J}^{-} u^{+}\right\|+C\left\|P_{-J}^{+} u^{+}\right\|
\end{gathered}
$$

with

$$
\varepsilon+\varepsilon^{\prime}<1 / 2
$$

If we admit this proposition, the conclusion of Theorem 3.3 is easily verified by the analogous argument to the one in the two dimensional case.

We turn to the proof of Proposition 6.1. We claim that for a $2 \times 2$ diagonal matrix $T=\operatorname{diag}\left[T_{1}, T_{2}\right]$ whose components are spherically symmetric and $T_{j}$ belong to $L^{\infty}\left(\mathbf{S}^{2}\right)$, we can easily verify the following result.

Lemma 6.2. For any positive integer $J$, it holds

$$
\left\|P_{J}^{+} T P_{-J}^{-}\right\| \leq C\left\|P_{-J}^{+}\right\|
$$

with a positive constant $C$.

From this and our assumption on $V$, it follows that

$$
\left\|P_{J}^{+}|x| V(x) P_{-J}^{-} u\right\| \leq \varepsilon\left\|P_{-J}^{-} u\right\|+C\left\|P_{-J}^{+} u\right\|
$$

with $\varepsilon<1 / 2$. 


\section{§7. The Negative Results}

Theorem 7.1. Suppose that $n=2$. There exist a positive constant $C>1$ and a (nontrivial) smooth function $u$ vanishing of infinite order at the origin of $\mathbb{R}^{2}$ such that

$$
|L u(x)| \leq \frac{C}{|x|}|u(x)| \quad \text { in } \mathbb{R}^{2}
$$

Proof of Theorem 7.1. As mentioned in the section 2, we will show that our result cannot be greatly improved.

First of all, we recall the result due to S. Alinhac and M. S. Baouendi. Let $\varepsilon$ be an arbitrarily small positive number. They constructed in [2] a nontrivial function $u_{0}(x) \in C^{\infty}\left(\mathbb{R}^{2}\right)$ which vanishes of infinite order at the origin of $\mathbb{R}^{2}$ and $a(x) \in C^{\infty}\left(\mathbb{R}^{2} \backslash\{0\}\right)$ satisfies

$$
\Delta u_{0}(x)+\frac{a(x)}{|x|^{2}} \partial_{\theta} u_{0}(x)=0,
$$

where

$$
\partial_{\theta}=x_{1} \frac{\partial}{\partial x_{2}}-x_{2} \frac{\partial}{\partial x_{1}}
$$

and $1+\varepsilon>|a(x)|>1$ in a neighborhood of the origin.

Using the polar coordinates $x=\left(x_{1}, x_{2}\right)=(r \cos \theta, r \sin \theta)$ and setting $y=$ $\log r$, we have

$$
\Delta u_{0}=e^{-2 y}\left(\partial_{y}^{2}+\partial_{\theta}^{2}\right) u_{0}=e^{-2 y} L_{+} L_{-} u_{0}=e^{-2 y} L_{-} L_{+} u_{0},
$$

where $L_{ \pm}=\partial_{y} \pm i \partial_{\theta}$. The equation (7.1) implies

$$
L_{+} L_{-} u_{0}+a\left(e^{y} \cos \theta, e^{y} \sin \theta\right) \frac{L_{+}-L_{-}}{2 i} u_{0}=0
$$

and

$$
L_{-} L_{+} u_{0}+a\left(e^{y} \cos \theta, e^{y} \sin \theta\right) \frac{L_{+}-L_{-}}{2 i} u_{0}=0 .
$$

On the other hand, the calculation in $\S 5$ shows that

$$
|x|\left(\sigma_{1} \partial_{1}+\sigma_{2} \partial_{2}\right)=\left(\begin{array}{cc}
0 & e^{-i \theta} L_{-} \\
e^{i \theta} L_{+} & 0
\end{array}\right) .
$$

Define $V(y, \theta)$ by

$$
\frac{a\left(e^{y} \cos \theta, e^{y} \sin \theta\right)}{2 i e^{y}}\left(\begin{array}{cc}
-e^{-i \theta} & e^{-i \theta} \\
-e^{i \theta} & e^{i \theta}
\end{array}\right)
$$


Then, it is easily verified that

$$
\left(\begin{array}{cc}
0 & e^{-i \theta} L_{-} \\
e^{i \theta} L_{+} & 0
\end{array}\right)\left(\begin{array}{l}
L_{-} u_{0} \\
L_{+} u_{0}
\end{array}\right)=V(x)\left(\begin{array}{c}
L_{-} u_{0} \\
L_{+} u_{0}
\end{array}\right) .
$$

In addition, we see that as the multiplication operator on $\mathbf{C}^{2}$

$$
\left\|e^{y} V(y, \theta)\right\|=\left|a\left(e^{y} \cos \theta, e^{y} \sin \theta\right)\right| .
$$

Returning to the original coordinates $\left(x_{1}, x_{2}\right)$, we obtain a desired function $u=$ ${ }^{t}\left(L_{-} u_{0}, L_{+} u_{0}\right)$.

Finally, we shall give another counterexample which shows that the result of Theorem 2.2 is optimal in the scale of the Lorentz spaces $L^{p, q}$ to which $\|V(x)\|$ of $(1.4)$ belongs. Let $n=3, \quad U={ }^{t}(u(x), 0,0,0)$, and $L=P_{1} \frac{\partial}{\partial x_{1}}+$ $P_{2} \frac{\partial}{\partial x_{2}}+P_{3} \frac{\partial}{\partial x_{3}}$, where

$$
P_{1}=\left(\begin{array}{cccc}
1 & 0 & 0 & 0 \\
0 & 1 & 0 & 0 \\
0 & 0 & -1 & 0 \\
0 & 0 & 0 & -1
\end{array}\right), \quad P_{2}=\left(\begin{array}{cccc}
0 & 0 & 1 & 0 \\
0 & 0 & 0 & -1 \\
1 & 0 & 0 & 0 \\
0 & -1 & 0 & 0
\end{array}\right), \quad P_{3}=\left(\begin{array}{cccc}
0 & 0 & 0 & 1 \\
0 & 0 & 1 & 0 \\
0 & 1 & 0 & 0 \\
1 & 0 & 0 & 0
\end{array}\right) .
$$

Then,

$$
L U(x)={ }^{t}\left(\frac{\partial u}{\partial x_{1}}(x), 0, \frac{\partial u}{\partial x_{2}}(x), \frac{\partial u}{\partial x_{3}}(x)\right)
$$

Let

$$
u(x)=\exp \left\{-(-\log |x|)^{1+\epsilon}\right\} .
$$

Then, $u(x)$ vanishes of infinite order at the origin of $\mathbf{R}^{3}$, and one can check that

$$
L U(x)=(1+\epsilon) e^{-(-\log |x|)^{1+\epsilon}} \frac{(-\log |x|)^{\epsilon}}{|x|}\left(\frac{x_{1}}{|x|}, 0, \frac{x_{2}}{|x|}, \frac{x_{3}}{|x|}\right) .
$$

Set

$$
W(x)=(1+\epsilon) \frac{(-\log |x|)^{\epsilon}}{|x|} .
$$

Then,

$$
|L U(x)|=W(x)|U(x)|
$$

and one can see that $W(x) \notin L_{l o c}^{3, \infty}\left(\mathbf{R}^{3}\right)$. In fact, a measurable function $f(x)$ on $\mathbb{R}^{n}$ belongs to $L_{l o c}^{3, \infty}\left(\mathbf{R}^{3}\right)$ if and only if for any compact set $K$ of $\mathbf{R}^{n}$ 


$$
\sup _{s>0} s|\{x \in K ;|f(x)|>s\}|^{1 / p}<+\infty
$$

where $|E|$ denotes the (Lebesgue) measure of $E$. (c.f. [12], p. 132).

\section{References}

[1] Alinhac, S. and Baouendi, M. S., Uniqueness for the characteristic Cauchy problem and strong unique continuation for higher order partial differential inequalities, Amer. J. Math., 102 (1980), 179-217.

[2] — A counterexample to strong uniqueness for partial differential equations of Schrödinger type, Comm. Partial Diff. Eqs., 19 (1994), 1727-1733.

[ 3 ] Aronszajn, N., Krzywcki, A. and Szarski, J., A unique continuation theorem for exterior differential forms on Riemanian manifolds, Ark. Mat., 4 (1962), 417-453.

[4] Grammatico, C., Unicitá forte per operatori ellittici, Tesi di Dottorato, Univ. degli Studi di Pisa (1997).

[ 5 ] Hile, G. N. and Protter, M. H., Unique continuation and the Cauchy problem for first order systems of partial differential equations, Comm. Partial Diff. Eqs., 1 (1976), 437-465.

[6] Jerison, D., Carleman inequalities for the Dirac and Laplace operator and unique continuation, Adv. Math., 63 (1986), 118-134.

[7] Jerison, D. and Kenig, C., Unique continuation and absence of positive eigenvalues for Schrödinger operators, Ann. of Math., 121 (1985), 463-492.

[ 8 ] Pan, Y. F., Unique continuation for Schrodinger operators with singular potentials, Comm. Partial Diff. Eqs., 17 (1992), 953-965.

[9] Regbaoui, R., Strong unique continuation results for differential inequalities, J. Funct. Anal., 148 (1997), 508-523.

[10] - Strong unique continuation for second order elliptic differential operators, J. Diff. Eqs., 141 (1997), 201-217.

[11] Thaller, B., The Dirac equation, Springer-Verlag 1992.

[12] Triebel, H., Interpolation Theory, Function Spaces, Differential operators, North-Holland Publishing Company, 1978.

[13] Vogelsang, V., Absence of embedded eigenvalues of the Dirac equation for long range potentials, Analysis, 7 (1987), 259-274.

[14] Wolff, T., A counterexample in a unique continuation problem, Comm. Anal. Geom., 2 (1994), 79-102.

[15] —, Recent work on sharp estimates in second order elliptic unique continuation problems, in Fourier Analysis and Partial Differential Equations, Garcia-Cuerva, Hernandez, Soria, Torrea ed., Stud. Adv. Math., CRC press (1995), 99-128. 